\title{
A Generalized Regression Neural Network Based on Soft Sensor for Multicomponent Distillation Column
}

\author{
Sanjay R. Patil ${ }^{*}$, V. N. Ghate ${ }^{2}$ \\ ${ }_{1}^{1}$ Department of Instrumentation Engineering, Government College of Engineering, Chandrapur, \\ Maharashtra, India. \\ ${ }^{2}$ Department of Electrical Engineering, Government College of Engineering, Chandrapur, Maharashtra, \\ India. \\ * Corresponding author. Tel.: 09423718206; email: sanjayrpatil_71@yahoo.com \\ Manuscript submitted October 20, 2014; accepted May 4, 2015. \\ doi: 10.17706/ijcce.2015.4.6.371-378
}

\begin{abstract}
Reliable and accurate measurement of product compositions is one of the main difficulties in distillation column control. In this paper a soft sensor based on generalized regression neural network (GRNN) is proposed to estimate the product composition of a multicomponent distillation column on the basis of simulated time series data. The results are compared with artificial neural network (ANN) based soft sensor. From the detailed dynamic simulation results, it is found that the proposed GRNN based estimator works better than ANN based soft sensor. The performance of estimator is evaluated in the presence of noise in the input.
\end{abstract}

Key words: Distillation column, generalized regression, neural network, soft sensor.

\section{Introduction}

In the monitoring and control of distillation columns, on-line composition measurements offer challenges. Unfortunately, no suitable hardware sensors are available in the market which could be used to measure required properties of various products on-line. The laboratory measurement procedures are tedious and time consuming. Hence there is a need for software-based sensors, which can estimate the product properties on-line, thus enabling effective control and optimization. Attempts have been made to estimate product properties using mathematical models. These models are so designed that they use some easily measurable secondary variables as inputs. However, the available models require much computation time or are not accurate enough to be used for feedback control [1].

In recent years, composition estimators using partial least squares (PLS) regression have been proposed [2]. ANN-based models have also been tried for this purpose [3]. While ANN modeling is a totally empirical approach, it can account for undefined non-linearities. Moreover once the ANN models are trained it takes very little time to predict the properties and hence is very suitable for on-line use. The development of ANN models requires expertise in the field of neural networks as well as the field in which the network is to be applied. There are several aspects such as the network architecture, set of inputs, training parameters, etc., the selection of which requires considerable attention and experience. In the past, a trial and error procedure has been used for designing a network which required up to several weeks for each product property.

Evolving Artificial Neural Network refer to a special class of ANN in which evolution is another 
fundamental form of adaptation in addition to learning. In this case, GA can be used to adapt connection weight, architecture design, learning rule adaptation, input feature selection and so on [2]. The evolution of ANN can increase model adaptability to a dynamic environment. In other words, the models developed were more robust to dynamic nonlinear process system [4]. This benefits the ANN since its generalization capability can be improved. In terms of architecture design and learning algorithm, GA can also help to reduce the uncertainties in some parameter selection.

The attempt to conjugate GA with ANN by the evolution of connection weights is discussed in our earlier paper [5]. The main aspect of neural networks design is the selection of training methods and corresponding parameters which has been widely studied [6]. Belew used genetic algorithm to assist in network training using backpropagation [7]. GA is used for optimally designing the network architecture as well as selecting weights and training rates [8]. Earlier also GA has been attempted for evolution of weights for large neural networks. Yao and Liu in 1997 had presented a new evolutionary system, i.e., EPNet to evolve ANN architecture and connection weights simultaneously [4].

The GRNN paradigm has been proposed as an alternative to the popular back-propagation training algorithm for feedforward neural networks. It is closely related to the probabilistic neural network. Regression can be thought of as the least- mean-squares estimation of the value of a variable based on available data. [9]

The principal advantages of GRNN are fast learning and convergence to the optimal regression surface as the number of samples becomes very large. GRNN is particularly advantageous with sparse data in a real-time environment, because the regression surface is instantly defined everywhere, even with just one sample. [10] The one sample estimate is that $Y$ will be the same as the one observed value regardless of the input vector $X$. A second sample will divide hyperspace into high and low halves with a smooth transition between them. The surface becomes gradually more complex with the addition of each new sample point.

\section{The Process}

The distillation column considered here is shown in Fig. 1. Where $F$ is feed consists of propane, $i$-butane, $n$-butane, $i$-pentane, $n$-pentane; $D$ is distillate; $B$ is bottoms; $C$ is condenser duty and $R$ is reboiler duty. It is a butane-pentane separator, the set of detailed specifications and operating conditions is given in Table 1 [11].

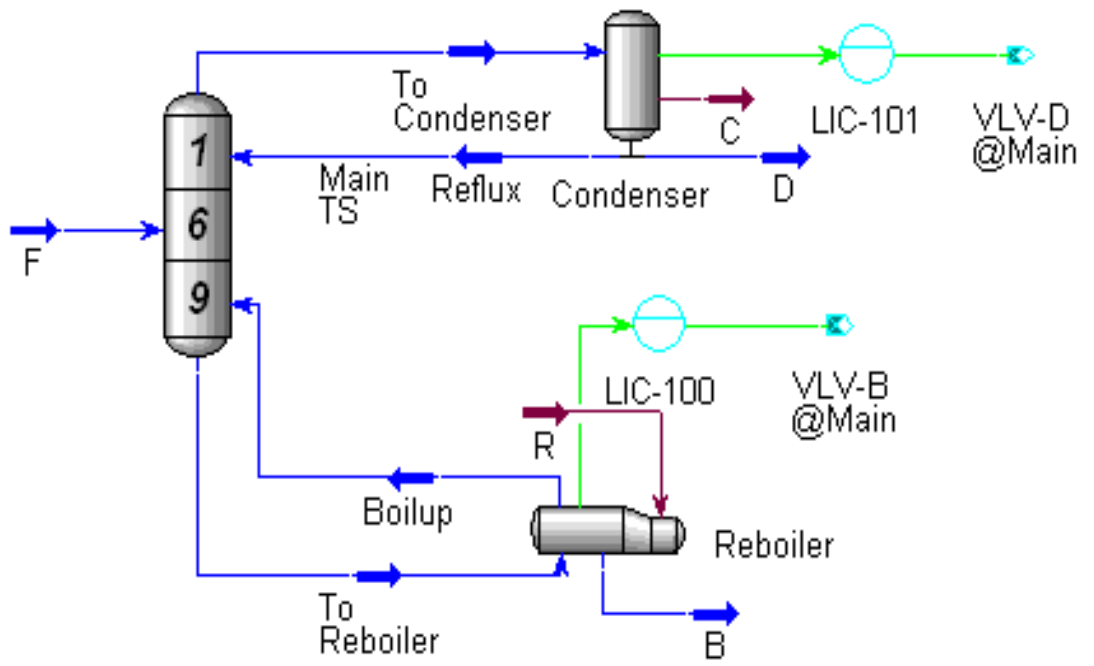

Fig. 1. Multicomponent distillation column.

AspenTech HYSYS plant simulation software is used to build the multicomponent distillation column, the 
Peng Robinson thermodynamic equations of state are used. Two level controllers are used one for condenser and another for reboiler which is essential for dynamic simulation. For the base case, the reflux ratio is 2.58. It is clear that increasing the reflux ratio has the desired effect of improving product purity. At lower distillate flow rates the mole fraction of heavy key ( $i$-pentane) in the distillate is small, and at higher distillate flow rates the mole fraction of light key ( $n$-butane) in the bottoms is small. Both cannot be small simultaneously. From this result we see that the "best" overall product purities are obtained when the distillate rate is in the vicinity of $45 \mathrm{lb} . \mathrm{mol} / \mathrm{h}$.

Table 1. Detailed Specifications and Operating Conditions for Distillation Column

\begin{tabular}{lcc}
\hline \hline Variable & Number & Value \\
\hline Number of stages & 1 & 9 \\
Feed stage location(F) & 1 & 6 \\
Component flows in feed & $c=5$ & $5,15,20,25,35 \mathrm{lb} . \mathrm{mol} / \mathrm{hr}$ \\
Feed pressure & 1 & $120 \mathrm{psia}$ \\
Feed vapor fraction & 1 & 0 \\
Pressure on each stage including condenser and reboiler & $N=11$ & $P_{j}=120 \mathrm{psia}$ \\
Heat duty on each stage except reboilers and condensers & $N-2=9$ & $Q_{j}=0$ \\
Vapor flow to condenser & 1 & $V_{2}=175 \mathrm{lb} . \mathrm{mol} / \mathrm{hr}$ \\
Distillate flow rate & 1 & $D=48.9 \mathrm{lb} . \mathrm{mol} / \mathrm{hr}$ \\
\hline \hline
\end{tabular}

\section{Data Generation and Preprocessing}

Process data that cover a wide range of operating conditions are necessary in the development of inferential estimators for product compositions. This work is based on simulation data obtained from HYSYS software. One of the advantages of using simulation data is that the data are free of measurement noises that may be present in real plant operation, although model mismatch is inevitable in simulations [12]. Another advantage of using simulation data is that the process data that covers a wide range of operating conditions can be easily obtained because changing operating conditions is easy in simulations.

The data generation here was done based on the correlation between primary and secondary variables. To obtain sufficiently excited data that cover the entire range of operating conditions, well planned step tests are required. Since the quality of data generated determines the validity of the resulting estimation model, we have carefully carried out the step tests. Here four selected input variables Feed flow rate 80 120 lb.mole/hr), Feed Temperature $\left(60-100^{\circ} \mathrm{F}\right)$, Reflux Ratio $(2-3)$ and Reboiler heat flow (1.5 - 2.3 $\mathrm{Btu} / \mathrm{hr}$ ) are used to generate sequence of random signals with varying amplitudes. This entire process was carried out within the HYSYS Plant environment and simulated for 3000 minutes. Initially steady state case was built and then dynamic simulation was carried out.

Data sampling interval was fixed at three minutes and thus a total amount of 1000 data were generated. The data pattern of input excitation signal sequence for above variables is shown in Fig. 2. Total 9 tray temperature variables and one distillate composition variable are generated using the step tests. The data profile for selected tray temperatures which were selected as model inputs for ANN and GRNN based estimators and corresponding distillate composition which is used as model output is shown in Fig. 3.

Neural network training can be made more efficient by performing certain preprocessing steps on the network inputs and targets (outputs). Before training, it is often useful to scale the inputs and targets so that they always fall within a specified range. The normalized inputs and targets are all fall in the interval $[-1,1]$ which is typical for tansig function in ANN. The MATLAB is used for these preprocessing tasks. 

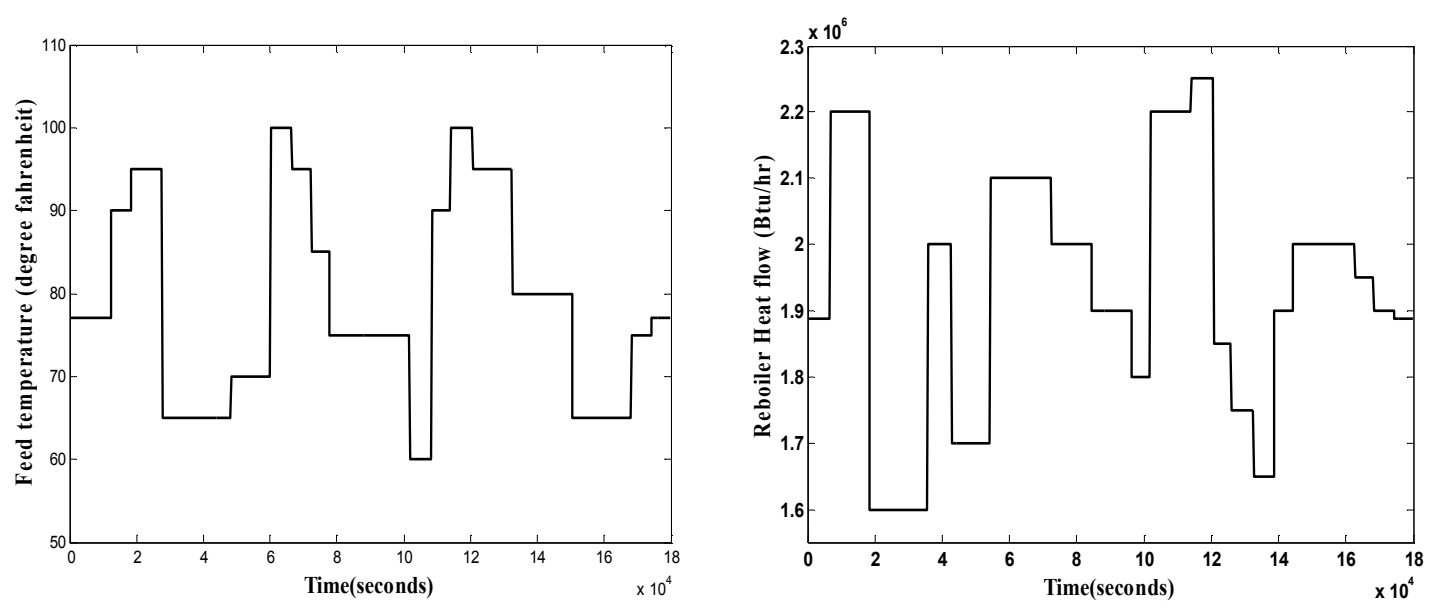

Fig. 2. Data profile of input variables.
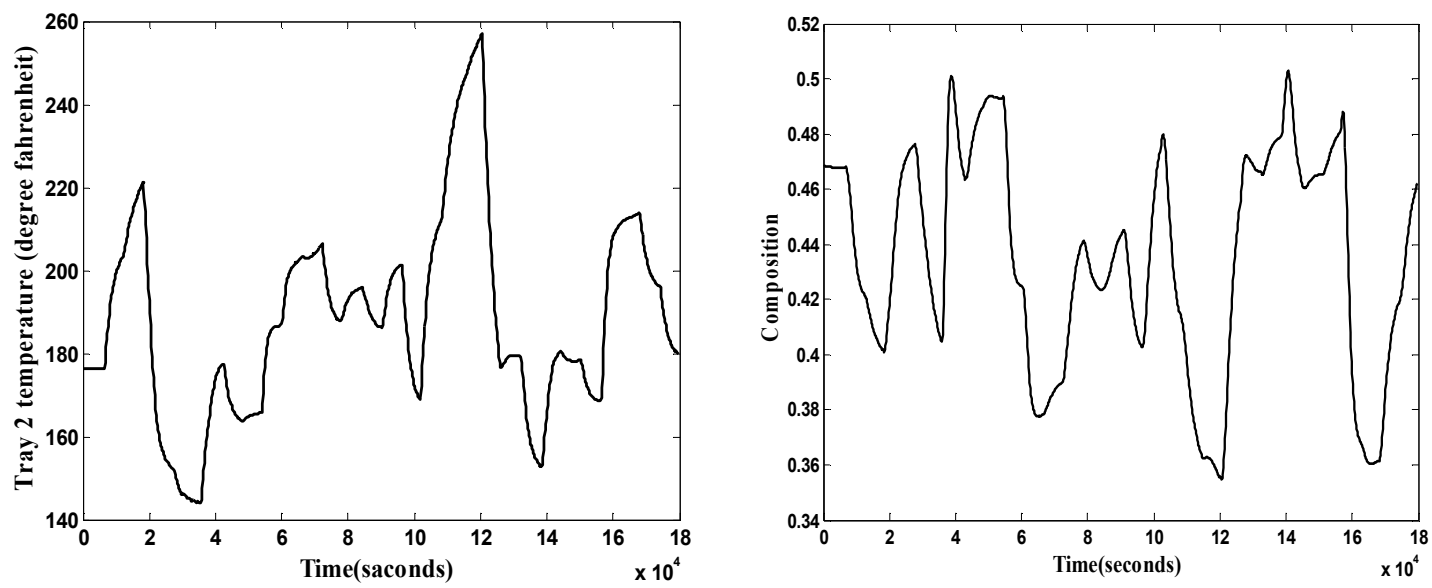

Fig. 3. Plots of temperature and composition.

\section{Soft Sensors}

\subsection{ANN Based Soft Sensor}

The associative property of artificial neural networks (ANN) and their inherent ability to learn and recognize highly non-linear and complex relationships finds them applications in engineering [13]. Given sufficient neurons in the hidden layer and a large set of input-output data to learn from, ANNs can approximate any continuous function arbitrarily well. Thus, ANN process models are more cost effective and eliminate the need for detailed effort. In developing the model, the network weights are adjusted to optimize a pre-set objective function (Mean square error). Since the ability to predict the dynamics of the unseen condition is of our interest, validation with different sets of data is performed [1]. In this study, the ANN model development efforts were carried out using the neural network toolbox within MATLAB.

There are several ways to evaluate the performance of estimator developed. The most important and the easiest way perhaps is by measuring the estimation accuracy. The estimation accuracy can be defined as the difference between the actual and estimated values. There are a number of approaches presenting the accuracy measures in the literature such as SSE (sum square error), RMSE (root mean square error), MAPE (mean absolute percentage error) and others. Most frequently used is the MSE (mean square error), defined as follow: 


$$
M S E=\frac{1}{N} \sum_{n=1}^{N}\left(y_{n}-\hat{y}_{n}\right)^{2}
$$

where $y$ is actual target value, $\hat{y}$ is estimated target value, and $N$ is total number of data.

In this study, trial and error approach was used to determine the optimum topology of the network. Starting from minimum number of neuron, the number of neurons in the hidden layer was increased upto 15 neurons in the network. Decision on the optimum topology was based on minimum error of validation, and it is observed that with 7 neurons in the hidden layer minimum MSE is obtained. Network trainings were accomplished using Levenberg-Marquadt algorithm. In this work, the dimension of the input vector is large, but the components of vectors are highly correlated. Hence it was required to reduce the dimension of the input vectors. An effective procedure for performing this operation is principal component analysis.

This technique has three effects: it orthogonalizes the components of the input vectors; it orders the resulting principal components so that those with the largest variation come first; and it eliminates those components that contribute the least to the variation in the data set. Note that we first normalize the input vectors; this is a standard procedure when using principal components [14].

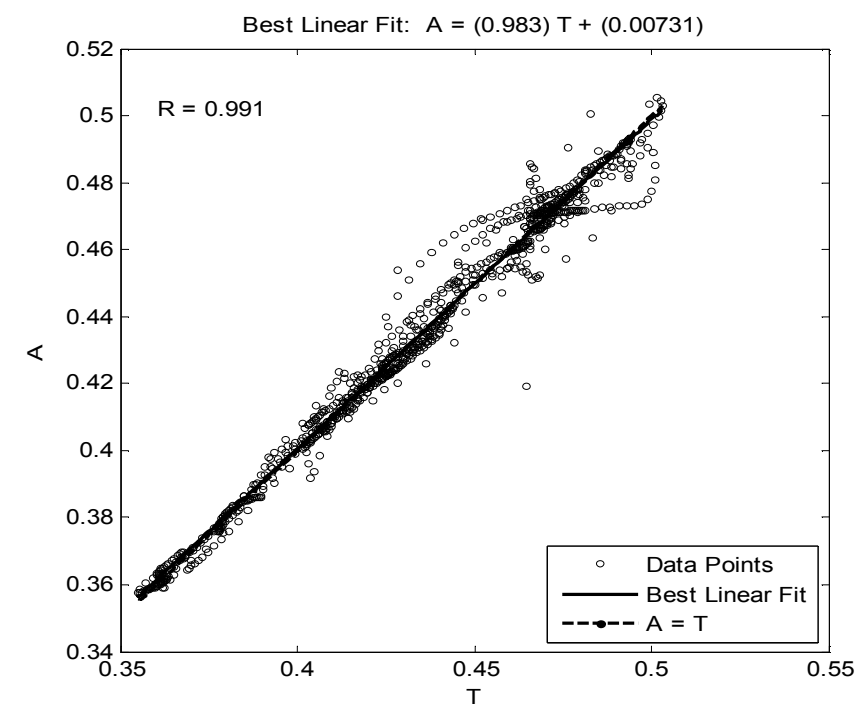

Fig. 4. Linear regression results.

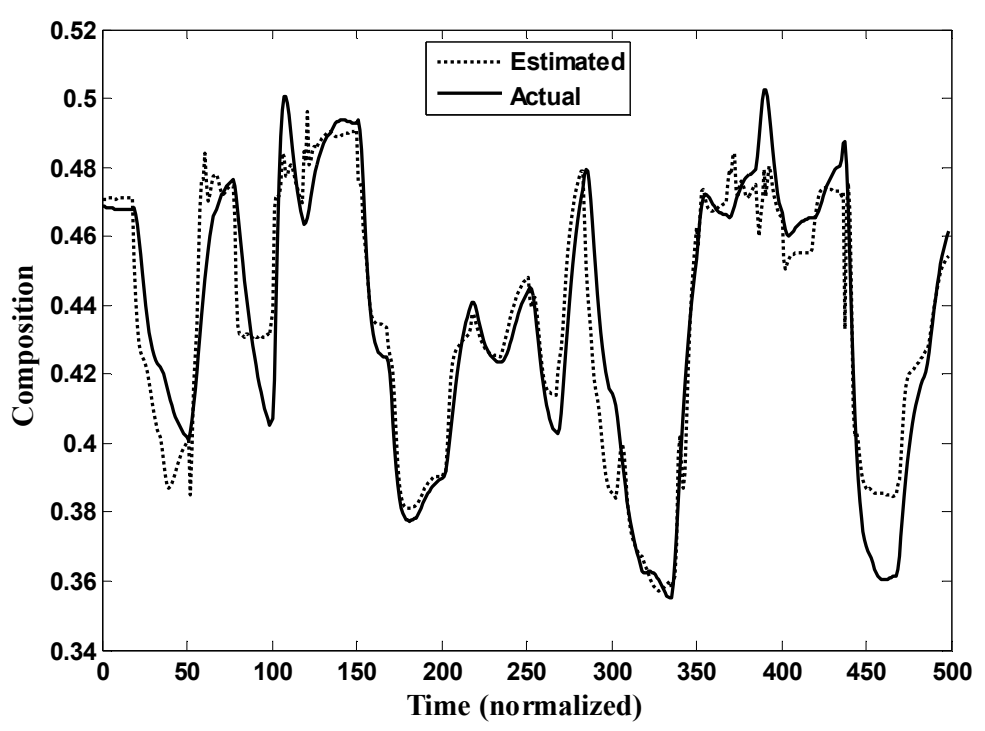

Fig. 5. Comparison of actual and estimated composition by ANN based soft sensor. 
Next the analysis of network response is carried out. This done by putting entire data set through the network and performed linear regression between network outputs and the corresponding targets. In this case, there is one output, so performed one regression, the result is shown in the Fig. 4. The R-value obtained is 0.983 which indicates that estimated output tracks the actual output reasonably well. Then actual and estimated outputs are compared by plotting them with respect to time (normalized), the results are presented in Fig. 5. The result shows that ANN based soft sensor gives good performance for estimation purpose.

\subsection{GRNN Based Soft Sensor}

A generalized regression neural network (GRNN) is often used for function approximation. It has a radial basis layer and a special linear layer. Unlike feedforward neural network its architecture does not include hidden layers, as mentioned above the first layer is radial basis layer where the distances between weight vector and input vector is considered to produce the net output.

The advantages of GRNN relative to other nonlinear regression techniques are as follows [10].

- The network "learns" in one pass through the data and can generalize from examples as soon as they are stored.

- The estimate converges to the conditional mean regression surfaces as more and more examples are observed; yet, as indicated in the examples, it forms very reasonable regression surfaces based on only a few samples.

- The estimate is bounded by the minimum and maximum of the observations.

- The estimate cannot converge to poor solutions corresponding to local minima of the error criterion (as sometimes happens with iterative techniques).

- The network can provide a mapping from one set of sample points to another. If the mapping is one to one, an inverse mapping can easily be generated from the same sample points.

The adjustment of 'spread' is important in the GRNN. The constructed GRNN soft sensor is trained for the input data by adjusting spread value to get the desired estimation accuracy. As spread becomes larger the radial basis function's slope becomes smoother and several neurons can respond to an input vector. The network then acts as if it is taking a weighted average between target vectors whose design input vectors are closest to the new input vector.

The comparison of MSE for both soft sensors is summarized in Table 2 and presented in Fig. 6.

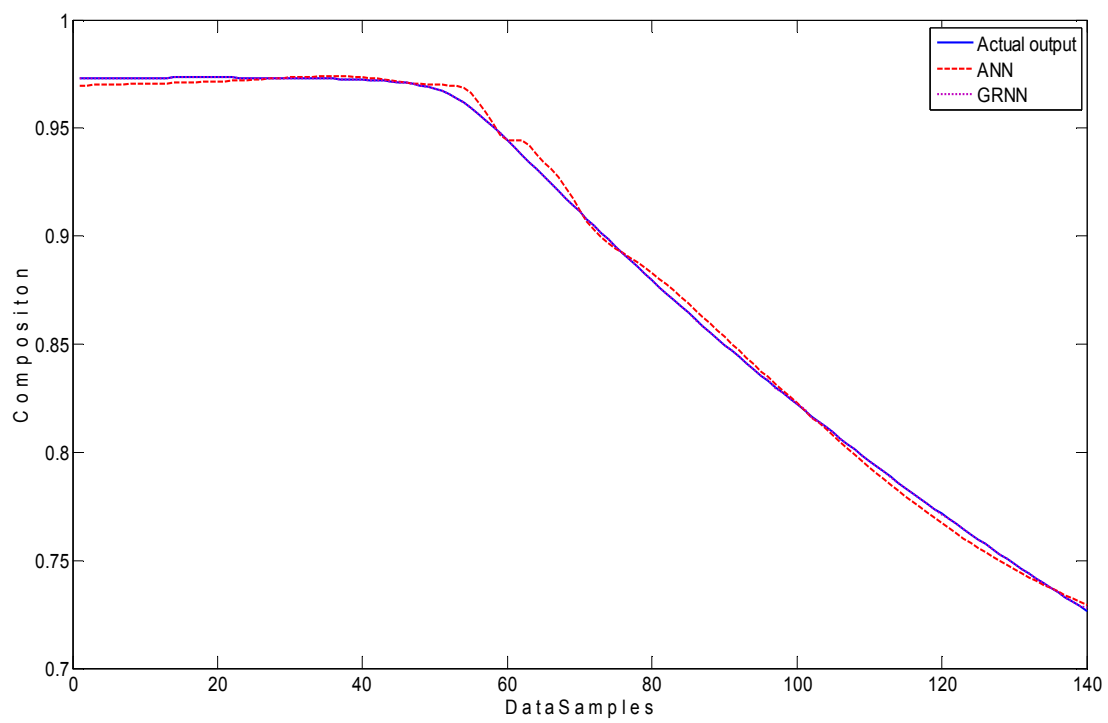

Fig. 6. Comparison of actual and estimated composition by ANN and GRNN soft sensors. 
Table 2. MSE Obtained for ANN and GRNN Based Soft Sensors

\begin{tabular}{lc}
\hline \hline Model & Mean Square Error (MSE) \\
\hline ANN based soft sensor & $1.282 \times 10^{-3}$ \\
GRNN based soft sensorr & $1.671 \times 10^{-6}$ \\
\hline \hline
\end{tabular}

\section{Conclusion}

In this study, soft sensors based on neural network and GRNN was developed for a simulated multicomponent distillation process in order to estimate the composition of the distillate stream using secondary process variables i.e. temperature measurements. Principal component analysis was used to analyze the available process data.

With respect to the characterization of the variables used as sensor inputs, it was evidenced that an effective composition estimation can be achieved even when not all of the available temperature measurements are used as input data to calibrate both soft sensors; on the contrary, the reduction of the number of temperatures in the input matrix does not necessarily deteriorate the estimation accuracy of the model. These results confirm the importance of proper data selection in the development of ANN and GRNN based soft sensors, and motivate further investigation in order to determine the optimal number and location of the temperature measurements to be used as soft sensor inputs.

The proposed GRNN soft sensor shows better performance than ANN soft sensor. Both the composition estimators are suitable for on-line use since they are tested on the dynamic time series data. Finally, the computing power required by the estimators is generally very low, which makes them attractive for on-line use.

\section{References}

[1] Patil, S. R., \& Nigam, M. J. (2009). Composition estimator as a soft sensor for distillation column using regression and neural network based models. Proceedings of Commune-09 (pp. 59-64). NIT, Kurukshetra.

[2] Chen, W. S. (2005). Application of artificial neural network, genetic algorithm in inferential estimation and control of a distillation column. M.E. Thesis, University Technology Malaysia.

[3] Singh, V., Gupta, I., \& Gupta, H. O. (2008). Inferential control of a distillation column using an online estimator. Chemical Product and Process Modeling, 3(1), 1934-2659.

[4] Yao, X., \& Liu, A. (1997). New evolutionary system for evolving artificial neural networks. IEEE Transactions on Neural Networks, 8(3), 694-713.

[5] Patil, S. R., \& Nigam, M. J. (2009). Soft sensor for Multicomponent distillation column using neural network and genetic algorithm based techniques. International Journal of Computational Intelligence Research, 5(3), 339-349.

[6] Dam, M., \& Saraf, D. N. (2006). Design of neural networks using genetic algorithm for on-line property estimation of crude fractionator products. Computers and Chemical Engineering, 30(4), 722-729.

[7] Belew, R. K., McInerney, J., \& Shraudolph, N. N. (1990). Evolving networks: Using the genetic algorithm with connectionist learning. CSE Technical Report\#CS90-174.

[8] Koehn, P. (1994). Combining genetic algorithms and neural networks: The encoding problem. M.S. Thesis, the University of Tennessee, Knoxville.

[9] Seng, T. L., Khalid, M., Yusof, R., \& Omatu, S. (1998). Adaptive neuro-fuzzy control system by RBF and GRNN neural networks. Journal of Intelligent and Robotic Systems, 23(2-4), 267-289.

[10] Specht, D. F. (1991). A general regression neural network. IEEE Transactions on Neural Networks, 2(6), 568-576. 
[11] Perry, R. H., \& Green, D. W. (2008). Perry's Chemical Engineers Handbook (8 ${ }^{\text {th }}$ ed.). McGraw-Hill, New York.

[12] Zamprogna, E., Barolo, M., \& Seborg, D. E. (2004). Estimating product composition profiles in batch distillation via partial lest squares regression. Control Engineering Practice, 12, 917-929.

[13] Osorio, D., Perez, J. R., Agosin, E., \& Cabrera, M. (2008). Soft sensor for on-line estimation of ethanol concentrations in wine stills. Journal of Food Engineering, 87, 571-577.

[14] Rajasekaran, S., \& Vijayalakshmi, G. A. (2004). Neural Networks, Fuzzy Logic and Genetic Algorithms (1 ${ }^{\text {st }}$ ed.). Prentice Hall of India, New Delhi.

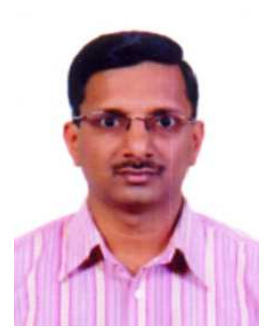

Sanjay R. Patil was born in Sangli, India in 1971. He received the B.E. degree in instrumentation engineering from Shivaji University, Kolhapur, in 1992 and the M.Tech. degree in control and guidance from Indian Institute of Technology, Roorkee, India, in 2009. From 1992 to 1994 he was with SNJP Polytechnic, Karnataka, from 1994 to 1997 he worked as a senior instrumentation engineer at Apex Industries and from 1997 to 2002 he was a faculty member at Dr. B. A. Technological University, Lonere, India. Since 2002 he is working with the Government College of Engineering, Directorate of Technical Education, Government of Maharashtra, India as an assistant professor in instrumentation. His areas of interest include process control, computational intelligence.

Sanjay R. Patil is a member of Instrument Society of India, Biomedical Society of India and the Indian Society for Technical Education (India).

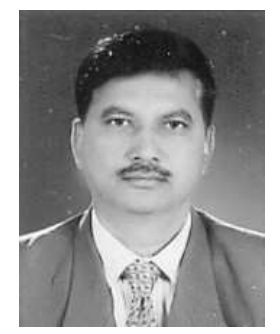

V. N. Ghate received the B.E. degree in electrical engineering from Sant Gadge Baba Amravati University, Amravati, India, in 1990, the M.E. degree in control systems from Shivaji University, Kolhapur, India, in 1993, and the Ph.D. degree from Sant Gadge Baba Amravati University in 2010. From 1994 to 2001, he was with the Department of Electrical Engineering, Government Polytechnic, Technical Education Government of Maharashtra. Since 2002, he has been with the Department of Electrical Engineering, Government College of Engineering, Technical Education Government of Maharashtra. His areas of interest include neural network, electrical machines and drives.

Dr. Ghate is a fellow of the Institution of Electronics and Telecommunication Engineers (India), Institution of Engineers (India) and a member of Instrument Society of India, IEEE and the Indian Society for Technical Education (India). 